\title{
Performance Analysis of MAC Protocols in Wireless Line Networks Using Statistical Mechanics
}

\author{
Ka Hung Hui, Dongning Guo and Randall Berry \\ Dept. of EECS, Northwestern Univ. \\ Evanston, IL 60208, USA
}

\author{
Martin Haenggi \\ Dept. of EE, Univ. of Notre Dame \\ Notre Dame, IN 46556, USA
}

\begin{abstract}
In this paper we consider the application of the Ising model from statistical mechanics to model interactions among interfering stations in a wireless network with a line topology. Each station employs a medium access control (MAC) protocol such that it decides to transmit or not depending on the states of its nearest neighbors and itself in the previous time slot. We use the Ising model to demonstrate how the interactions between neighboring stations affect the one-hop throughput of the network and to optimize the underlying MAC protocol. Both the cases of single-packet and multipacket reception are considered. For single-packet reception, the throughput achieved by an optimized protocol is shown to be $15.8 \%$ better than that achieved by slotted ALOHA. In the multipacket reception case, the MAC protocol achieves the same throughput as TDMA, which doubles the throughput of slotted ALOHA.
\end{abstract}

\section{INTRODUCTION}

The performance of wireless networks is limited by mutual interference between stations. Because the power of a signal attenuates with distance, interference is largely a shortrange effect among 'neighboring' stations. Most existing medium access control (MAC) protocols such as ALOHA and Carrier-Sense Multiple Access (CSMA) exploit this phenomenon and attempt to distributedly schedule stations so that at most one station in a local neighborhood transmits at a given time. It has been recognized that the resulting interactions among stations in a wireless network is similar to that found in the Ising model [1], [2] in statistical mechanics (see for example [3]). In this paper, we will explore this connection in depth for a wireless line network.

The Ising model was originally used to model a spin system in stationarity, where a spin system consists of a number of spins on a regular lattice. The spins can be in 'up' or 'down' states, and their states are affected by an external magnetic field acting on them and the interactions between neighboring spins. For our purposes, the spins in the system will model the stations in a wireless network, where the 'up' and 'down' states represent whether a station is transmitting or idle, respectively. We will begin by considering a timeslotted MAC protocol in which each station observes the states of its neighboring stations in the previous time slot and then probabilistically decides its state in the current time slot based only on the observed states of its neighbors and its own state in the previous time slot. For example, the well-known slotted ALOHA protocol can be viewed as a special case of this model in which a station simply transmits in each slot with a fixed probability, regardless of its observation.
For the resulting model, we consider the following two important questions:

- Validity of the Ising model: The network evolving according to this type of protocol can be described by a Markov field in space and a (first-order) Markov chain in time. Does this Markov chain admit a stationary distribution? If the stationary distribution exists, will it be a Markov field? That is, does the stationary distribution only consist of short-range interactions as in an Ising model?

- Performance Issue: How do we define the interactions between neighboring stations to optimize the performance of the network, e.g., the transmission probability or the throughput?

We consider a wireless line network in which each station broadcasts packets to its two nearest neighbors. The channel can be either a collision channel or a multipacket reception channel [4]. Our contributions include the following:

1) We use Markov Chain of Markov Fields (MCMF) [5], [6] to show that under mild conditions (reversible and synchronous, defined in Section IV) this type of MAC protocol leads to a stationary state such that only short-range interactions exist in the stationary state, which implies the validity of using the Ising model to represent a wireless network.

2) We analytically derive the throughput and transmission probability of a wireless network using techniques in statistical mechanics, explicitly demonstrating the effect of interactions between neighboring stations on these performance measures.

3) We show that if a station chooses its current state only based on the states of its neighbors in the previous time slot, then the performance is the same as slotted ALOHA, regardless of whether the channel is a collision channel or a multipacket reception channel.

4) If a station chooses its current state based on the states of its neighbors and its own state in the previous time slot, we show that the performance is strictly better than slotted ALOHA: for the collision channel, there is an increase of $15.8 \%$ in the optimal throughput and a reduction of $11.83 \%$ in transmission probability to achieve the optimal throughput; while for the multipacket reception channel, the throughput can be doubled while keeping the same transmission 
probability, which is the same performance as TDMA.

The rest of the paper is organized as follows. Section II reviews related works. We describe the MAC protocol in detail and discuss the condition for the protocol to lead to a stationary state having short-range interactions in Sections III, IV and V. In Section VI, we derive the transmission probability and throughput of the wireless network using techniques in statistical mechanics, and interpret them in Section VII. Section VIII concludes and discusses future works.

\section{RELATED WORKS}

A variety of tools from statistical mechanics have been used to study the interactions between stations in wireless networks. In [3] a similar Markov chain model was used to model the interactions among stations employing an idealized CSMA protocol without collision. However, in [3] the spins represented the links between stations, while here we use them to represent the stations themselves and consider a more general class of MAC protocols. In [7] the totally asymmetric simple exclusion process (TASEP) model from statistical mechanics was used to study the throughput and end-to-end delay of a single unidirectional data flow in a multihop wireless line network.

Mean field theory is another technique in statistical mechanics that has been applied to wireless networks. For example, [8] used mean field arguments to prove that the decoupling assumption used in [9] for evaluating the performance of IEEE 802.11 Distributed Coordination Function is asymptotically exact as the number of stations in the network grows. However, mean field arguments are not invariably correct [2]. In this work, we use the Ising model to address the exact interactions between neighbors rather than approximating the interactions using mean field theory.

Another common technique from statistical mechanics is identifying phase transitions. For example, this was used in [10] to study the connectivity properties of large random networks and in [11] to consider possible activations of sets of interfering links in wireless networks on regular lattices.

\section{MARKov Chain of MARKov Fields (MCMF) MODEL FOR MAC PROTOCOLS}

We now describe the network model we will study. We consider a wireless line network with $N$ stations, denoted by $S=\{1,2, \ldots, N\}$. Each station broadcasts packets to its two nearest neighbors. We assume every station is saturated, so that queueing is not considered.

The state of each station assumes values in $E=$ $\{+1,-1\}$, where +1 and -1 represent the station transmitting or staying idle, respectively. Let $\Omega=E^{S}$ denote the configuration space, i.e., the state space of the entire network. Let $X_{i}(t) \in E$ be the state of station $i$ at time $t$, and $\mathbf{X}(t)=\left\{X_{i}(t)\right\}_{i \in S} \in \Omega$ be the configuration, or the state of the network, at time $t$. We are interested in MAC protocols for which $\mathbf{X}=\{\mathbf{X}(t)\}_{t \in \mathbb{N}}$ is a Markov Chain of Markov Field (MCMF) [6], i.e., a process for which

- $\mathbf{X}(1), \mathbf{X}(2), \ldots$ is a Markov chain on $\Omega$, and
- for every $t, X_{1}(t), \ldots, X_{N}(t)$ is a Markov field on $\Omega$ conditioned on $\mathbf{X}(t-1)$.

Under such a MAC protocol, each station can choose its current state based on the previous states of all stations in the network. For example, let $\mathbf{x}=\left\{x_{i}\right\}_{i \in S}$ and $\mathbf{y}=\left\{y_{i}\right\}_{i \in S}$ be the previous and current configurations respectively. Station $i$ observes the previous states of stations $i-1, i, i+1$ and chooses its current state according to

$p_{i}\left(y_{i} \mid \mathbf{x}\right) \propto \exp \left(h_{i} y_{i}+J_{i, i-1} x_{i-1} y_{i}+J_{i, i} x_{i} y_{i}+J_{i, i+1} x_{i+1} y_{i}\right)$.

The term $h_{i} y_{i}$ in (1) is the instantaneous interaction potential and is in general denoted by $\phi_{i}(\mathbf{y})$. It controls how aggressive station $i$ attempts transmissions, independent of everything else. In this example, a positive $h_{i}$ favors transmission and a negative $h_{i}$ suppresses transmission. The terms having the form of $J_{i, j} x_{j} y_{i}$ in (1) are the conditional interaction potentials and are in general denoted by $\phi_{i, j}(\mathbf{y}, \mathbf{x})$. They control how station $i$ chooses its current state based on the observed state of station $j$ in the previous time slot. In this example, station $i$ tends to choose the same state as station $j$ in the previous time slot if $J_{i, j}$ is positive, and does the opposite if $J_{i, j}$ is negative. The potentials $\phi_{i}$ and $\phi_{i, j}$ can be defined arbitrarily, as long as the following two conditions are satisfied: $(i) \phi_{i}(\mathbf{y})=\phi_{i}\left(y_{i}\right)$ only depends on $y_{i}$, and (ii) $\phi_{i, j}(\mathbf{y}, \mathbf{x})=\phi_{i, j}\left(y_{i}, x_{j}\right)$ only depends on $y_{i}$ and $x_{j}$. In general, define the conditional energy $U(\mathbf{y} \mid \mathbf{x})$ as

$$
U(\mathbf{y} \mid \mathbf{x})=\sum_{i \in S}\left(\phi_{i}\left(y_{i}\right)+\sum_{j \in S} \phi_{i, j}\left(y_{i}, x_{j}\right)\right),
$$

the transition probability of the Markov chain, $P(\mathbf{y} \mid \mathbf{x})=$ $\operatorname{Pr}\{\mathbf{X}(t)=\mathbf{y} \mid \mathbf{X}(t-1)=\mathbf{x}\}$, is then given by

$$
P(\mathbf{y} \mid \mathbf{x})=\frac{\exp U(\mathbf{y} \mid \mathbf{x})}{\sum_{\mathbf{z} \in \Omega} \exp U(\mathbf{z} \mid \mathbf{x})}
$$

The transition probability in this form has the nice property of being synchronous (defined in the following section). Also, it is easy to control the relative strength of the potentials. For example, if the previous state of a station is twice as important as the previous states of its neighbors, we can represent this by defining the potentials so that $\left|\phi_{i, i}\left(y_{i}, x_{i}\right)\right|=2\left|\phi_{i, i-1}\left(y_{i}, x_{i-1}\right)\right|=2\left|\phi_{i, i+1}\left(y_{i}, x_{i+1}\right)\right|$.

In a wireless network, it is possible that station $i$ cannot observe the state of station $j$ in the previous time slot due to their physical separation. To model this, for all $i$, let $V_{i}$ be the set of neighbors of station $i$ and let $\phi_{i, j}=0$ if $j \notin V_{i}$. In other words, the summation over $j \in S$ in (2) should be replaced by a summation over $j \in V_{i}$.

\section{Markov Stationary Behavior of MCMF}

Next we investigate when the Markov chain defined in the previous section has a stationary distribution with the Markov property. In [6] it is shown that a sufficient condition for this to occur is that the Markov chain is time-reversible and the transition probability is synchronous. We consider these two properties in the following. 
The transition probability $P(\mathbf{y} \mid \mathbf{x})$ is said to be synchronous if for all $\mathbf{x}$ and $\mathbf{y}$ there are $\left\{p_{i}\left(y_{i} \mid \mathbf{x}\right)\right\}_{i \in S}$ such that

$$
P(\mathbf{y} \mid \mathbf{x})=\prod_{i \in S} p_{i}\left(y_{i} \mid \mathbf{x}\right), p_{i}\left(y_{i} \mid \mathbf{x}\right)>0, \text { and } \sum_{u \in E} p_{i}(u \mid \mathbf{x})=1 .
$$

The transition probability is synchronous if, given $\mathbf{x}$ as the state of the network in the previous time slot, each station chooses its current state independently and at the same time with distributions $\left\{p_{i}(\cdot \mid \mathbf{x})\right\}_{i \in S}$ over $E$. For the model defined in Section III, the transition probability is synchronous because

$$
\begin{aligned}
P(\mathbf{y} \mid \mathbf{x}) & =\frac{\exp U(\mathbf{y} \mid \mathbf{x})}{\sum_{\mathbf{z} \in \Omega} \exp U(\mathbf{z} \mid \mathbf{x})} \\
& =\frac{\exp \sum_{i \in S}\left(\phi_{i}\left(y_{i}\right)+\sum_{j \in S} \phi_{i, j}\left(y_{i}, x_{j}\right)\right)}{\sum_{\mathbf{z} \in \Omega} \exp \sum_{i \in S}\left(\phi_{i}\left(z_{i}\right)+\sum_{j \in S} \phi_{i, j}\left(z_{i}, x_{j}\right)\right)} \\
& =\frac{\prod_{i \in S} \exp \left(\phi_{i}\left(y_{i}\right)+\sum_{j \in S} \phi_{i, j}\left(y_{i}, x_{j}\right)\right)}{\prod_{i \in S} \sum_{z_{i} \in E} \exp \left(\phi_{i}\left(z_{i}\right)+\sum_{j \in S} \phi_{i, j}\left(z_{i}, x_{j}\right)\right)} \\
& =\prod_{i \in S} p_{i}\left(y_{i} \mid \mathbf{x}\right),
\end{aligned}
$$

where

$$
p_{i}\left(y_{i} \mid \mathbf{x}\right)=\frac{\exp \left(\phi_{i}\left(y_{i}\right)+\sum_{j \in S} \phi_{i, j}\left(y_{i}, x_{j}\right)\right)}{\sum_{z_{i} \in E} \exp \left(\phi_{i}\left(z_{i}\right)+\sum_{j \in S} \phi_{i, j}\left(z_{i}, x_{j}\right)\right)}
$$

is a valid probability distribution because both $\phi_{i}\left(y_{i}\right)$ and $\phi_{i, j}\left(y_{i}, x_{j}\right)$ depend on $\mathbf{y}$ through $y_{i}$ only. Note that (4) is the probability that station $i$ chooses state $y_{i}$ in the protocol described in Section III.

From [5], [6], the Markov chain is time-reversible if $\phi_{i, j}\left(y_{i}, x_{j}\right)=\phi_{j, i}\left(x_{j}, y_{i}\right)$ for all $i, j, y_{i}, x_{j}$. This means that the effect of station $j$ in the previous time slot on station $i$ in the current time slot is the same as the effect of station $i$ in the previous time slot on station $j$ in the current time slot. Therefore, by [6] the MAC protocol described in Section III admits a stationary distribution with Markov property if $\phi_{i, j}\left(y_{i}, x_{j}\right)=\phi_{j, i}\left(x_{j}, y_{i}\right)$ for all $i, j, y_{i}, x_{j}$.

As noted in [12], the stationary distribution is in general difficult to compute, and introducing reversibility is mainly for mathematical convenience. Hence, we will assume the reversibility condition holds in the rest of the paper.

\section{MARKOV PROPERTY OF MCMF IN RELATION TO INTERACTION POTENTIALS}

In this section we discuss how the Markov property of the stationary distribution of a MCMF $\mathbf{X}$ is related to its interaction potentials $\phi_{i}$ and $\phi_{i, j}$.

Let $-\mathbf{1} \in \Omega$ be the configuration in which all stations are in state -1 . We use this as a reference state. Let $X_{i}$ be the random variable representing the state of station $i$ in stationarity. Since the Markov chain is time-reversible, the stationary distribution $\pi(\mathbf{y})=\operatorname{Pr}\left\{X_{i}=y_{i}, \forall i \in S\right\}$ for any configuration $\mathbf{y} \in \Omega$ satisfies the detailed balance condition:

$$
\pi(\mathbf{y}) P(-\mathbf{1} \mid \mathbf{y})=\pi(-\mathbf{1}) P(\mathbf{y} \mid-\mathbf{1}) .
$$

Therefore, from (3),

$$
\begin{aligned}
\pi(\mathbf{y}) & =\pi(-\mathbf{1}) \frac{P(\mathbf{y} \mid-\mathbf{1})}{P(-\mathbf{1} \mid \mathbf{y})} \\
& =\pi(-\mathbf{1}) \frac{\exp U(\mathbf{y} \mid-\mathbf{1})}{\sum_{\mathbf{z} \in \Omega} \exp U(\mathbf{z} \mid-\mathbf{1})} \frac{\sum_{\mathbf{z} \in \Omega} \exp U(\mathbf{z} \mid \mathbf{y})}{\exp U(-\mathbf{1} \mid \mathbf{y})} \\
& \propto \exp (U(\mathbf{y} \mid-\mathbf{1})-U(-\mathbf{1} \mid \mathbf{y})) \sum_{\mathbf{z} \in \Omega} \exp U(\mathbf{z} \mid \mathbf{y})
\end{aligned}
$$

Since $\phi_{i, j}\left(y_{i}, x_{j}\right)=\phi_{j, i}\left(x_{j}, y_{i}\right)$ for all $i, j, y_{i}, x_{j}$, by switching the order of summation and then interchanging $i$ with $j$, we get

$$
\begin{aligned}
& \sum_{i \in S} \sum_{j \in S} \phi_{i, j}\left(y_{i},-1\right)-\sum_{i \in S} \sum_{j \in S} \phi_{i, j}\left(-1, y_{j}\right) \\
= & \sum_{i \in S} \sum_{j \in S} \phi_{j, i}\left(-1, y_{i}\right)-\sum_{i \in S} \sum_{j \in S} \phi_{i, j}\left(-1, y_{j}\right) \\
= & \sum_{j \in S} \sum_{i \in S} \phi_{j, i}\left(-1, y_{i}\right)-\sum_{i \in S} \sum_{j \in S} \phi_{i, j}\left(-1, y_{j}\right) \\
= & \sum_{i \in S} \sum_{j \in S} \phi_{i, j}\left(-1, y_{j}\right)-\sum_{i \in S} \sum_{j \in S} \phi_{i, j}\left(-1, y_{j}\right) \\
= & 0,
\end{aligned}
$$

we have from (2)

$$
\begin{aligned}
& \exp (U(\mathbf{y} \mid \mathbf{- 1})-U(\mathbf{- 1} \mid \mathbf{y})) \\
= & \exp \left(\sum_{i \in S} \phi_{i}\left(y_{i}\right)-\sum_{i \in S} \phi_{i}(-1)\right. \\
& \left.+\sum_{i \in S} \sum_{j \in S} \phi_{i, j}\left(y_{i},-1\right)-\sum_{i \in S} \sum_{j \in S} \phi_{i, j}\left(-1, y_{j}\right)\right) \\
\propto & \exp \sum_{i \in S} \phi_{i}\left(y_{i}\right) .
\end{aligned}
$$

Also, from (2),

$$
\begin{aligned}
& \sum_{\mathbf{z} \in \Omega} \exp U(\mathbf{z} \mid \mathbf{y}) \\
= & \sum_{\mathbf{z} \in \Omega} \exp \sum_{i \in S}\left(\phi_{i}\left(z_{i}\right)+\sum_{j \in S} \phi_{i, j}\left(z_{i}, y_{j}\right)\right) \\
= & \prod_{i \in S} \sum_{z_{i} \in E} \exp \left(\phi_{i}\left(z_{i}\right)+\sum_{j \in S} \phi_{i, j}\left(z_{i}, y_{j}\right)\right) \\
= & \exp \sum_{i \in S} \ln \sum_{z_{i} \in E} \exp \left(\phi_{i}\left(z_{i}\right)+\sum_{j \in S} \phi_{i, j}\left(z_{i}, y_{j}\right)\right) .
\end{aligned}
$$


Combining (5)-(7), we obtain

$$
\pi(\mathbf{y}) \propto \exp \sum_{i \in S} \Phi_{i}(\mathbf{y})
$$

where

$$
\Phi_{i}(\mathbf{y})=\phi_{i}\left(y_{i}\right)+\ln \sum_{z_{i} \in E} \exp \left(\phi_{i}\left(z_{i}\right)+\sum_{j \in S} \phi_{i, j}\left(z_{i}, y_{j}\right)\right) \text {. }
$$

For short-range interactions, (9) can be reduced to

$$
\Phi_{i}(\mathbf{y})=\phi_{i}\left(y_{i}\right)+\ln \sum_{z_{i} \in E} \exp \left(\phi_{i}\left(z_{i}\right)+\sum_{j \in V_{i}} \phi_{i, j}\left(z_{i}, y_{j}\right)\right) \text {. }
$$

Define

$$
\begin{aligned}
W_{i}=\{j \in S \backslash\{i\}: & \exists k \in S \text { such that } i, j \in V_{k}, \\
& \text { or } j \in V_{i} \text { if } \phi_{i} \neq 0, \\
& \text { or } \left.i \in V_{j} \text { if } \phi_{j} \neq 0\right\},
\end{aligned}
$$

meaning that $j \in W_{i}$ if for some $k \in S, \Phi_{k}(\mathbf{y})$ depends on both $y_{i}$ and $y_{j}$. If there is a $k$ such that both $i$ and $j$ are neighbors of $k$, then both $y_{i}$ and $y_{j}$ appear in $\Phi_{k}(\mathbf{y})$. If $\phi_{i} \neq 0$, then $\Phi_{i}(\mathbf{y})$ already depends on $y_{i}$, therefore if $j \in$ $V_{i}$, both $y_{i}$ and $y_{j}$ appear in $\Phi_{i}(\mathbf{y})$. Then in stationarity, if $j \in W_{i}, X_{j}$ and $X_{i}$ will be coupled. Therefore, the stationary distribution of $X_{i}$ conditioned on $\left\{X_{j}\right\}_{j \in S \backslash\{i\}}$ only depends on $\left\{X_{j}\right\}_{j \in W_{i}}$.

The set $V_{i} \backslash\{i\}$ represents the stations (except station $i$ ) having temporal dependence with station $i$, while the set $W_{i}$ represents the stations having dependence with station $i$ in stationarity. It is noted that $V_{i} \backslash\{i\}$ and $W_{i}$ are not the same in general. The condition that $V_{i} \backslash\{i\} \subset W_{i}$ does not necessarily hold. This is illustrated by the following two examples:

1) Assume $V_{i}=\{i-1, i, i+1\}$ for all $i \in S$. Then $y_{i}$ appears in $\Phi_{i-1}(\mathbf{y}), \Phi_{i}(\mathbf{y}), \Phi_{i+1}(\mathbf{y})$. Therefore, $W_{i}=$ $\{i-2, i-1, i+1, i+2\} \neq V_{i} \backslash\{i\}$ and $V_{i} \backslash\{i\} \subset$ $W_{i}$. The stationary distribution of $X_{i}$ conditioned on $\left\{X_{j}\right\}_{j \in S \backslash\{i\}}$ depends on $X_{i-1}, X_{i+1}$, and also $X_{i-2}, X_{i+2}$.

2) Assume $V_{i}=\{i-1, i+1\}$ for all $i \in S$. In addition, suppose $\phi_{i}=0$ for all $i \in S$. Then $y_{i}$ only appears in $\Phi_{i-1}(\mathbf{y})$ and $\Phi_{i+1}(\mathbf{y})$. In this case, $W_{i}=\{i-2, i+2\} \neq V_{i} \backslash\{i\}$ and $V_{i} \backslash\{i\} \not \subset$ $W_{i}$. Therefore, in stationarity, the distribution of $X_{i}$ conditioned on $\left\{X_{j}\right\}_{j \in S \backslash\{i\}}$ depends on $X_{i-2}, X_{i+2}$, but not $X_{i-1}, X_{i+1}$. In this case, the system effectively decouples into two independent sub-systems in stationarity, one consisting of all odd numbered stations and the other consisting of all even numbered stations.

In general, the interaction range in stationarity doubles the interaction range defined by the protocol.

\section{Vi. Performance Analysis using Statistical MECHANICS}

Next we turn to analyzing the steady-state performance of a MAC protocol defined according to (1). For ease of exhibition we restrict ourselves to the following two cases:
1) $h_{i}=h$ for all $i, V_{i}=\{i-1, i, i+1\}$ for all $i, J_{i, i-1}=$ $J_{i, i+1}=J$ and $J_{i, i}=J^{\prime}$ for all $i$,

2) $h_{i}=h$ for all $i, V_{i}=\{i-1, i+1\}$ for all $i, J_{i, i-1}=$ $J_{i, i+1}=J$ for all $i$.

In Case 1, the state of a station depends on the previous states of its nearest neighbors and its own previous state, while in Case 2, the state of a station depends on the previous states of its nearest neighbors only. Note that Case 2 is a subset of Case 1 with $J^{\prime}=0$. Hence, in the following we will only describe Case 1 unless otherwise stated.

The stationary distribution defined by these potentials is

$$
\pi(\mathbf{y})=\frac{1}{Z} \exp \sum_{i \in S} \Phi_{i}(\mathbf{y})
$$

where from (10)

$$
\Phi_{i}(\mathbf{y})=h y_{i}+\ln \cosh \left[h+J\left(y_{i-1}+y_{i+1}\right)+J^{\prime} y_{i}\right],
$$

and

$$
Z=\sum_{\mathbf{y} \in \Omega} \exp \sum_{i \in S} \Phi_{i}(\mathbf{y})
$$

Therefore, we can consider the stationary distribution as an Ising model [1] with Hamiltonian

$$
H(\mathbf{y})=-\sum_{i \in S} \Phi_{i}(\mathbf{y})
$$

and partition function

$$
Z=\sum_{\mathbf{y} \in \Omega} e^{-H(\mathbf{y})}
$$

To calculate any steady-state performance measure, we have to first evaluate the partition function. To do this, we can use the transfer matrix approach in statistical mechanics [1]. Define the transfer matrix $\mathbf{V}$ as in (15). The rows from top to bottom and the columns from left to right correspond to states $(+1,+1),(+1,-1),(-1,+1)$, and $(-1,-1)$ respectively. The entries in $\mathbf{V}$ then represent the transition from the 'row state' $\left(y_{i-1}, y_{i}\right)$ to the 'column state' $\left(y_{i}, y_{i+1}\right)$. If we assume a periodic boundary condition, i.e., the ends of the network are joined such that the stations at both ends are neighbors (the stations form a Markov field), then

$$
Z=\operatorname{trace} \mathbf{V}^{N}=\lambda_{1}^{N}+\lambda_{2}^{N}+\lambda_{3}^{N}+\lambda_{4}^{N},
$$

where $\lambda_{1}, \lambda_{2}, \lambda_{3}, \lambda_{4}$ are the eigenvalues of $\mathbf{V}$. The only eigenvalue that we need in the following calculations is $\lambda_{1}$, where we assume it to be the eigenvalue having the maximum magnitude, which is always real, positive and unique by the Perron-Frobenius Theorem [13]. This eigenvalue $\lambda_{1}$ admits analytical solution as the largest (in amplitude) root of the fourth-order characteristic polynomial of the transfer matrix. The solution is straightforward but omitted here due to the large length of the expression. It can be shown that in the special case of $J^{\prime}=0$,

$$
\lambda_{1}=\frac{1}{2}\left(e^{2 J} \cosh 2 h+e^{-2 J}+2 \sqrt{1+e^{4 J} \sinh ^{2} h} \cosh h\right) \text {. }
$$




$$
\mathbf{V}=\left(\begin{array}{cccc}
e^{h} \cosh \left(h+2 J+J^{\prime}\right) & e^{h} \cosh \left(h+J^{\prime}\right) & 0 & 0 \\
0 & 0 & e^{-h} \cosh \left(h+2 J-J^{\prime}\right) & e^{-h} \cosh \left(h-J^{\prime}\right) \\
e^{h} \cosh \left(h+J^{\prime}\right) & e^{h} \cosh \left(h-2 J+J^{\prime}\right) & 0 & 0 \\
0 & 0 & e^{-h} \cosh \left(h-J^{\prime}\right) & e^{-h} \cosh \left(h-2 J-J^{\prime}\right)
\end{array}\right)
$$

We are interested in the following performance measures of a wireless network: transmission probability and throughput. The network is translation invariant in both cases, meaning that the expectations of any $X_{i}$ are the same, and the correlations among any set of $X_{i}$ 's only depend on them via the differences between their positions in the network. This is because the corresponding potentials are invariant under translations. The transmission probability $p$ is the expected number of transmission attempts of a station in a time slot, i.e.,

$$
p=\mathbb{E}\left[\frac{1}{2}\left(1+X_{i}\right)\right]=\frac{1}{2}\left(1+\mathbb{E}\left[X_{i}\right]\right) .
$$

We define the throughput $\rho$ as the expected number of packets received successfully by a station from its neighbors in a time slot. We assume each transmission can only reach its nearest neighbors. The expression for the throughput depends on whether the underlying channel is a collision channel or a multipacket reception channel. For the collision channel, if the interference range and the transmission range are the same, then a station can receive a packet when it is idle and only one of its neighbors is transmitting. Hence,

$$
\begin{aligned}
\rho_{\mathrm{cc}}= & \mathbb{E}\left[\frac{1}{8}\left(1-X_{i-1}\right)\left(1-X_{i}\right)\left(1+X_{i+1}\right)\right. \\
& \left.+\frac{1}{8}\left(1+X_{i-1}\right)\left(1-X_{i}\right)\left(1-X_{i+1}\right)\right] \\
= & \frac{1}{4}\left(1-\mathbb{E}\left[X_{i}\right]-\mathbb{E}\left[X_{i-1} X_{i+1}\right]+\mathbb{E}\left[X_{i-1} X_{i} X_{i+1}\right]\right) .
\end{aligned}
$$

For the multipacket reception channel, we assume that each station can receive two packets at the same time, then as long as a station is idle, the number of packets it receives is equal to the number of neighbors transmitting, which means there is no interference. Then,

$$
\begin{aligned}
\rho_{\mathrm{mpr}} & =\mathbb{E}\left[\frac{1}{4}\left(1+X_{i-1}\right)\left(1-X_{i}\right)+\frac{1}{4}\left(1-X_{i}\right)\left(1+X_{i+1}\right)\right] \\
& =\frac{1}{2}\left(1-\mathbb{E}\left[X_{i} X_{i+1}\right]\right) .
\end{aligned}
$$

Therefore we have to calculate $\mathbb{E}\left[X_{i}\right], \mathbb{E}\left[X_{i} X_{i+1}\right]$, $\mathbb{E}\left[X_{i-1} X_{i+1}\right]$ and $\mathbb{E}\left[X_{i-1} X_{i} X_{i+1}\right]$.

To calculate $\mathbb{E}\left[X_{i}\right]$ for example, we can make the following modifications [14]: add $\alpha y_{i}$ to $\Phi_{i}(\mathbf{y})$ defined in (12), define the corresponding transfer matrix $\mathbf{V}_{\alpha}$, compute its largest eigenvalue $\lambda_{\alpha, 1}$, then

$$
\mathbb{E}\left[X_{i}\right]=\left.\frac{\partial \ln \lambda_{\alpha, 1}}{\partial \alpha}\right|_{\alpha=0} .
$$

The other expectations can be computed similarly. The main difficulty in this approach is that the expression for $\lambda_{\alpha, 1}$ can be complicated. Here, we introduce a method to compute these expectations using
1) $\chi_{\alpha}(\lambda)$, the characteristic polynomial of $\mathbf{V}_{\alpha}$,

2) $\chi(\lambda)$, the characteristic polynomial of $\mathbf{V}$, and

3) $\lambda_{1}$, the largest eigenvalue of $\mathbf{V}$.

This provides a simpler way to calculate $\left.\frac{\partial \ln \lambda_{\alpha, 1}}{\partial \alpha}\right|_{\alpha=0}$, since $\chi_{\alpha}(\lambda)$ is easier to compute than $\lambda_{\alpha, 1}$. Also, if we want to compute the expectation of another quantity, we only have to compute a new $\chi_{\alpha}(\lambda)$ accordingly and reuse the other two. This method is based on the following proposition.

Proposition 1: Let $\mathbf{V}_{\alpha}$ be a square matrix parametrized by a variable $\alpha, \chi_{\alpha}(\lambda)$ be the characteristic polynomial of $\mathbf{V}_{\alpha}$ and $\lambda_{0,1}$ be the eigenvalue of $\mathbf{V}_{0}$ with multiplicity one. If $\mathbf{V}_{\alpha}$ is differentiable at $\alpha=0$, then

$$
\left.\frac{\partial \ln \lambda_{\alpha, 1}}{\partial \alpha}\right|_{\alpha=0}=-\frac{\left.\frac{\partial \chi_{\alpha}(\lambda)}{\partial \alpha}\right|_{\lambda=\lambda_{0,1}, \alpha=0}}{\left.\lambda_{0,1} \frac{\partial \chi_{0}(\lambda)}{\partial \lambda}\right|_{\lambda=\lambda_{0,1}}},
$$

where $\lambda_{\alpha, 1}$ is the eigenvalue of $\mathbf{V}_{\alpha}$ such that it is differentiable at $\alpha=0$ and $\lim _{\alpha \rightarrow 0} \lambda_{\alpha, 1}=\lambda_{0,1}$.

Proof: If $\mathbf{V}_{\alpha}$ is differentiable at $\alpha=0$, then $\chi_{\alpha}(\lambda)=$ $\operatorname{det}\left(\mathbf{V}_{\alpha}-\lambda \mathbf{I}\right)$ is differentiable with respect to $\alpha$ at $\alpha=0$, and the existence of $\lambda_{\alpha, 1}$ with the stated properties when $\alpha$ is in the vicinity of 0 is guaranteed by [15]. Then (19) follows upon differentiating $\chi_{\alpha}\left(\lambda_{\alpha, 1}\right)=0$ with respect to $\alpha$ at $\alpha=0$.

The transfer matrix $\mathbf{V}_{\alpha}$ defined before Proposition 1 is differentiable with respect to $\alpha$ at $\alpha=0, \mathbf{V}$ in (15) can be taken as $\mathbf{V}_{0}$ in Proposition 1 and $\lambda_{1}$ is an eigenvalue of V with multiplicity one as mentioned above by the PerronFrobenius Theorem, therefore Proposition 1 applies. Also, $\lambda_{\alpha, 1}$ in Proposition 1 is the eigenvalue of $\mathbf{V}_{\alpha}$ with the largest magnitude, as a consequence of the Perron-Frobenius Theorem. Therefore, we can calculate the expectations simply using Proposition 1. In Case 2, the $\lambda_{0,1}$ in Proposition 1 is given by (14).

Following this approach, we get the following for Case 2:

$$
\begin{aligned}
p & =\frac{e^{2 J} \sinh h+\sqrt{1+e^{4 J} \sinh ^{2} h}}{2 \sqrt{1+e^{4 J} \sinh ^{2} h}}, \\
\rho_{\mathrm{cc}} & =\frac{e^{-h} \lambda_{1} \cosh (h-2 J)-\sinh ^{2} 2 J}{2 \lambda_{1}^{2}\left(1+e^{4 J} \sinh ^{2} h\right)}, \\
\rho_{\mathrm{mpr}} & =\frac{1}{2\left(1+e^{4 J} \sinh ^{2} h\right)} .
\end{aligned}
$$

Contour plots of transmission probability and throughput against $h$ and $J$ for the collision channel and the multipacket reception channel for Case 2 are shown in Figs. 1(a), 1(b) and 1(c) respectively. For Case 1, the expressions are too complicated and are omitted for clarity. Figs. 2, 3(a) and 


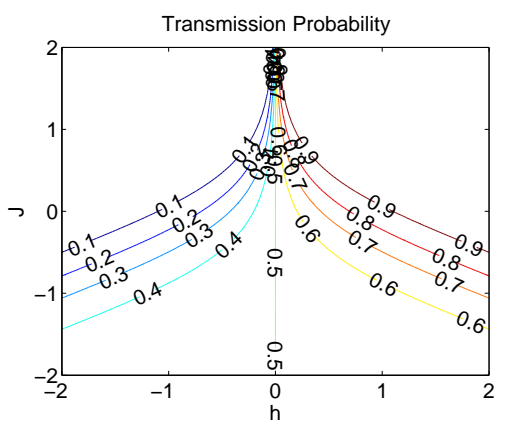

(a)

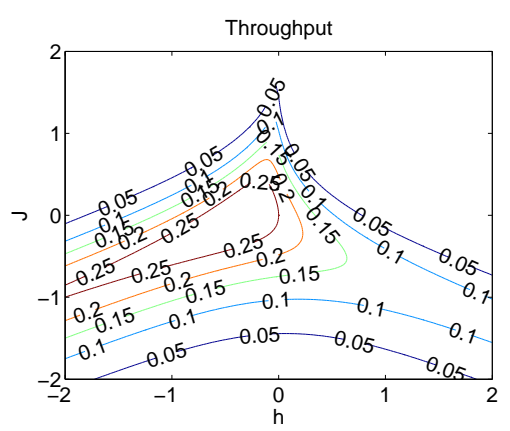

(b)

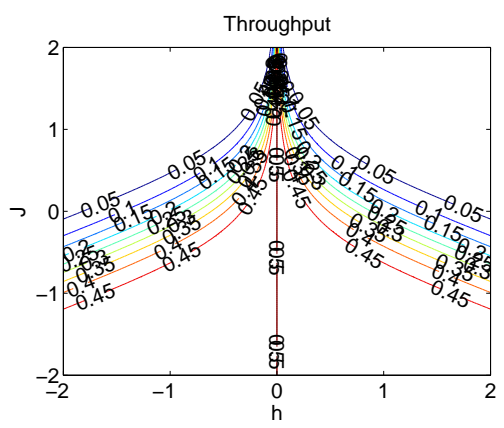

(c)

Fig. 1. Performance for Case 2: (a) transmission probability, (b) throughput in the collision channel, and (c) throughput in the multipacket reception channel.

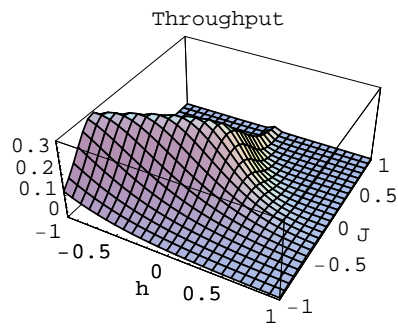

Fig. 2. Throughput at $J^{\prime}=4$ in the collision channel (Case 1).

3(b) are some plots of the throughput in the collision channel and the multipacket reception channel showing the maximum throughput. Figs. 3(c) and 3(d) show the transmission probability corresponding to the throughput shown in Fig. 3(b).

\section{AnAlysis of The Results}

We divide this section into four parts. We discuss the collision channel first and then the multipacket reception channel. For both channels, we first discuss the more general Case 1 in Section VI and then Case 2.

\section{A. Collision Channel for Case 1}

We first discuss the effects of $h, J$ and $J^{\prime}$ on the transmission probability. First suppose $J>0$. If $J>0$, when a station transmitted in the previous time slot, its neighbors will have a higher probability to transmit in the current time slot; and if a station was idle in the previous time slot, its neighbors will have a higher probability to be idle in the current time slot. Therefore, when $h>0$, every station has a high tendency to transmit, $J>0$ will result in all stations attempting transmissions with high probability. On the contrary, when $h<0$, every station has a high tendency to be idle, $J>0$ will keep the stations in remaining silence with high probability. Hence, the transmission probability is close to 1 when $J>0, h>0$ and close to 0 when $J>0, h<0$. The transmission probability changes abruptly as $h$ moves across 0 while keeping $J>0$ fixed, and the change is more rapid for larger $J$. If $J^{\prime}>0$, each station tends to remain in the same state, which reinforces the effect of $h$ on the transmission probability; while if $J^{\prime}<0$, each station tends to switch between different states, counteracting the effect of $h$ on the transmission probability. Therefore, the effect of $J^{\prime}$ on the transmission probability is to control how abrupt the change of the transmission probability is when $h$ moves across 0 .

Suppose now $J<0$. When $h>0$, each station has a high tendency to transmit, but as $J<0$, a station will tend to remain idle as its neighbors transmitted in the previous time slot. These two factors counteract each other, resulting in each station attempting transmissions with approximately half probability. Therefore, no matter whether $J^{\prime}$ is positive or negative, each station remains transmitting with half probability on average. Similar statements can be made for the case of $J<0, h<0$.

Next we discuss how the throughput varies with $h, J$ and $J^{\prime}$. When $J>0$, either all stations are aggressive in transmissions $(h>0)$ or all stations tend to remain idle $(h<0)$. In both cases, the throughput is very low due to excessive collision or underutilization of the wireless channel. Also, in order to have a high throughput, $J$ and $J^{\prime}$ should have opposite signs so that the previous state of a station has opposite effects on the current state of itself and its neighbors. Therefore when $J>0$, the throughput can be high only if $h \lesssim 0$ and $J^{\prime}<0$.

When $J<0$, if a station was transmitting, its neighbors will tend to be idle in the current time slot; and if a station was idle, its neighbors will tend to attempt transmissions in the current time slot. Therefore, the two nearest neighbors of a station always tend to choose the same action. This means that either there is collision or nothing is transmitted in a slot with high probability. Hence, the throughput is also very low. However, this argument only applies when $J^{\prime}<0$. When $J^{\prime}>0$, the optimal throughput can be achieved.

The optimal throughput does not correspond to a single $\left(h, J, J^{\prime}\right)$ tuple: the throughput is at maximum when $J^{\prime}$ is sufficiently large, both $h$ and $J$ are sufficiently negative with $h \gtrsim 2 J$. The optimal throughput is 0.3431 and the corresponding transmission probability is 0.2939. This is illustrated in Fig. 2. The optimal values of $h, J$ and $J^{\prime}$ can be explained by (12). Since $h<0$, the first term of (12) suppresses transmission to reduce excessive collisions. For 


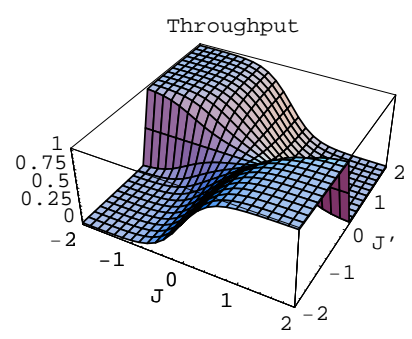

(a)

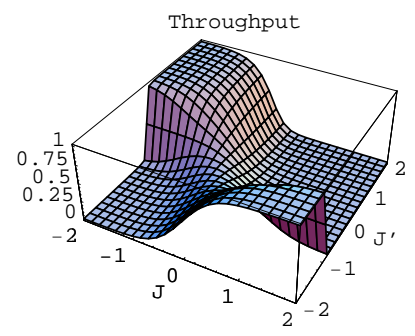

(b)

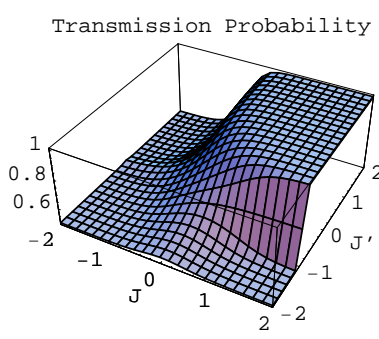

(c)

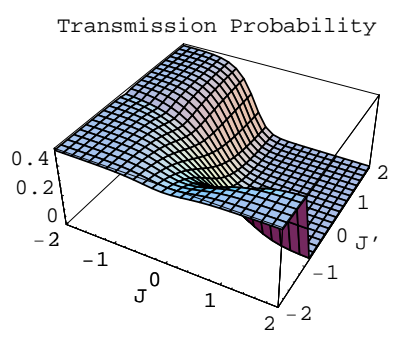

(d)

Fig. 3. Performance for Case 1 in the multipacket reception channel: (a) throughput at $h=0$, (a) throughput at $|h|=1$, (c) transmission probability at $h=1$, and (d) transmission probability at $h=-1$.

the second term, we divide into three cases:

- $y_{i-1}=-y_{i+1}$ : There is already one neighbor transmitting, it is better for a station to remain idle, otherwise it cannot receive from its neighbor. In this case, $J\left(y_{i-1}+\right.$ $\left.y_{i+1}\right)=0$ and $h+J\left(y_{i-1}+y_{i+1}\right)+J^{\prime} y_{i}$ has a larger magnitude when $y_{i}=-1$, which helps in suppressing transmission.

- $y_{i-1}=y_{i+1}=+1$ : Both neighbors are transmitting, therefore it is better for a station to remain idle, since even if it attempts a transmission, both neighbors cannot receive. In this case, $h+J\left(y_{i-1}+y_{i+1}\right)<0$ and $h+$ $J\left(y_{i-1}+y_{i+1}\right)+J^{\prime} y_{i}$ has a larger magnitude when $y_{i}=-1$, which also helps in suppressing transmission.

- $y_{i-1}=y_{i+1}=-1$ : In this case $h+J\left(y_{i-1}+y_{i+1}\right) \gtrsim 0$ and therefore $h+J\left(y_{i-1}+y_{i+1}\right)+J^{\prime} y_{i}$ has a larger magnitude when $y_{i}=+1$, which makes transmissions a bit more likely to occur than the other cases. As both neighbors are idle, it is possible that they can both receive the packet successfully.

In the collision channel, if we use TDMA, the optimal throughput is $\frac{2}{3}$. To achieve that throughput, one in every three stations must transmit, i.e., a pattern of repeating $+1,-1,-1$ should be observed in the network. This shows that the nearest neighbor interactions alone are not effective in maintaining the repeating $+1,-1,-1$ pattern in the network.

We can also illustrate why the interaction range doubles in stationarity with this example. We define the throughput to be the expected number of successful receptions at a station. For station $i$ to receive a packet successfully, it must be idle and only one of the stations $i-1$ and $i+1$ is transmitting. Since whether station $i-1$ transmits or not depends on the states of both stations $i$ and $i-2$, and whether station $i+1$ transmits or not depends on the states of stations $i$ and $i+2$, whether station $i$ receives a packet successfully will depend on stations $i-2, i-1, i+1, i+2$.

\section{B. Collision Channel for Case 2}

If we set $J^{\prime}=0$, the optimal throughput is $\rho_{\mathrm{cc}}=\frac{8}{27}$, which occurs at $J=0, h=-\frac{1}{2} \ln 2$. At the optimal $h$ and
$J$, the corresponding $p$ is

$$
\frac{e^{h}}{e^{h}+e^{-h}}=\frac{1}{3} .
$$

This is also the optimal throughput for slotted ALOHA in the collision channel: Suppose $p$ is the transmission probability of a station, the throughput is then $2 p(1-p)^{2}$. The optimal throughput can be found by optimizing over all $p$, and the optimal throughput is $\frac{8}{27}$ with the corresponding $p$ as $\frac{1}{3}$. The probability that a transmission results in a successful reception is therefore $\frac{8 / 27}{2 \cdot 1 / 3}=\frac{4}{9}$.

Comparing with the results for Case 1 in Section VII-A, we can see that with a nonzero $J^{\prime}$, there is a $15.8 \%$ improvement in the optimal throughput and a $11.83 \%$ reduction in the corresponding transmission probability. The probability that a transmission results in a successful reception with a nonzero $J^{\prime}$ is $\frac{0.3431}{2 \cdot 0.2939}=0.5837$, which shows a $31.33 \%$ improvement over the results for $J^{\prime}=0$.

\section{Multipacket Reception Channel for Case 1}

In the multipacket reception channel, there is no collision, hence the dependence of throughput on $h, J$ and $J^{\prime}$ exhibits different behaviors.

The throughput can be arbitrarily close to 1 , for example, by choosing $h=0$ with $J$ and $J^{\prime}$ having opposite signs and with sufficiently large magnitudes. This is illustrated in Fig. 3(a). Suppose $J^{\prime}>0$. Then a station tends to stay in the same state. Therefore, with $J<0$, its neighbors will tend to choose the opposite state and stay in that state. On the contrary, suppose $J^{\prime}<0$. A station tends to switch between both states. With $J>0$, its neighbors in the current time slot will tend to choose the same state as the station did in the previous time slot, which is the opposite state chosen by the station in the current time slot. In both cases, the configurations of alternating +1 and -1 are the most probable ones. The difference between these two cases is that in the first one, a station captures the channel and transmits continuously, while in the second one, stations share the channel by taking turn in transmitting.

From Fig. 3(a), we observe that when $J^{\prime}$ deviates slightly from 0 , the throughput can change abruptly to either 1 or 0 for sufficiently large $J$. This means as $J^{\prime}$ is not zero, the dependence of the previous and current states of the 
same station across time helps in aligning stations so that all neighboring stations are either in opposite states or in the same state.

When $h$ is not zero, similar relationships between the throughput, $J$ and $J^{\prime}$ are also observed, except that the abrupt change in throughput occurs at $J^{\prime}=0$ for negative $J$ and $J^{\prime}=-|h|$ for positive $J$, and the change is more rapid for larger $|h|$. An example is shown in Fig. 3(b) for $|h|=1$.

For any $\left(h, J, J^{\prime}\right)$ tuple such that the throughput is around one, the corresponding transmission probability is always around a half. Therefore the probability that a transmission results in a successful reception is around one, which can only happen when neighboring stations are in opposite states.

In the multipacket reception channel, if we use TDMA, the optimal throughput is 1 . In this case, one in every two stations transmits, i.e., a pattern of alternating +1 and -1 should be observed. Indeed, this pattern appears if we choose $h, J, J^{\prime}$ appropriately. This shows that the nearest neighbor interactions can be used to maintain the alternating +1 and -1 pattern in the network, which implies this is a distributed way to implement TDMA in the multipacket reception channel. Together with the results in the collision channel, we can observe the dependence of the length of the activity pattern and the interaction range of the MAC protocol.

\section{Multipacket Reception Channel for Case 2}

Consider the case where $J^{\prime}=0$. Suppose $J>0$. When $h<0$, the throughput is low due to underutilization of the wireless channel. When $h>0$, the throughput is also low simply because all stations are aggressive in transmissions and therefore with low probability the stations are in idle state for receiving packets. On the contrary, suppose $J<0$. The throughput can be arbitrarily close to $\frac{1}{2}$ by choosing a sufficiently negative $J$. With such $J$, each station transmits with approximately half probability. If a station is idle in the previous time slot, its neighbors tend to transmit in the current time slot. Since the state of a station is independent of the state of itself in the previous time slot, in the current time slot it may be receiving or transmitting with half probability. Then the throughput will be about $2 \cdot \operatorname{Pr}\{$ a station is idle in the previous time slot $\}$. $\operatorname{Pr}\{$ a station is idle in the current time slot $\}=2 \cdot \frac{1}{2} \cdot \frac{1}{2}=\frac{1}{2}$.

The optimal throughput in the multipacket reception channel is $\rho_{\mathrm{mpr}}=\frac{1}{2}$, which occurs at $h=0$ and $J$ can be any value. The corresponding $p$ is $\frac{1}{2}$. This is also the optimal throughput for slotted ALOHA in the multipacket reception channel: Suppose $p$ is the transmission probability of a station, the throughput is then $2 p(1-p)$. The optimal throughput in this case is $\frac{1}{2}$ with the corresponding $p$ as $\frac{1}{2}$.

Together with the results in Section VII-B, we can see that to optimize the throughput, a nonzero $J^{\prime}$ should be used.

\section{CONCLUSION}

In this paper, we studied a MAC protocol in which each station chooses its current state based on the states of its neighbors and itself in the previous time slot, and proposed using the Ising model in statistical mechanics to obtain the transmission probability and throughput of wireless networks in the collision channel and the multipacket reception channel. Our results showed that, if each station decides to transmit based on the observed states of its neighbors only, the best possible performance is the same as slotted ALOHA. However, if each station makes its decision based on the states of its neighbors and itself in the previous time slot, then in the collision channel the throughput can be increased while the corresponding transmission probability can be reduced; and for the multipacket reception channel, the throughput can be doubled while keeping the same transmission probability, which is the same performance as TDMA.

The results obtained here assumed the protocol is executed for a long time such that the network is already in stationarity. One possibility for future works is to determine the convergence time for the protocol. We assumed symmetry in the mutual interactions between stations to get the results. Another direction would be to consider the case that there is no symmetry in the interactions.

\section{REFERENCES}

[1] R. J. Baxter, Exactly Solved Models in Statistical Mechanics. Dover, 2007.

[2] M. Plischke and B. Bergersen, Equilibrium Statistical Physics, 3rd ed. World Scientific, 2006.

[3] M. Durvy, O. Dousse, and P. Thiran, "On the fairness of large CSMA networks," IEEE J. Sel. Areas Commun., Sep. 2009, to appear.

[4] S. Ghez, S. Verdú, and S. C. Schwartz, "Stability properties of slotted ALOHA with multipacket reception capability," IEEE Trans. Autom. Control, vol. 30, no. 7, pp. 640-649, Jul. 1988.

[5] X. Guyon, Random Fields on a Network: Modeling, Statistics and Applications. Springer-Verlag, 1995.

[6] X. Guyon and C. Hardouin, "Markov chain markov field dynamics: Models and statistics," Statistics, vol. 36, no. 4, pp. 339-363, 2002.

[7] S. Srinivasa and M. Haenggi, "TASEPs: A statistical mechanics tool to analyze the performance of wireless line networks," IEEE/ACM Trans. Netw., 2009, submitted to.

[8] C. Bordenave, D. McDonald, and A. Proutière, "Random multiaccess algorithms - a mean field analysis," in Allerton Conference on Communication, Control and Computing, Monticello, IL, USA, Sep. 2005, pp. 494-503.

[9] G. Bianchi, "Performance analysis of the IEEE 802.11 distributed coordination function," IEEE J. Sel. Areas Commun., vol. 18, no. 3, pp. 535-547, Mar. 2000.

[10] B. Krishnamachari, S. B. Wicker, and R. Béjar, "Phase transition phenomena in wireless ad hoc networks," in Globecom, San Antonio, TX, USA, Nov. 2001.

[11] M. B. Hastings, "Statistical mechanics of interfering links," Physical Review E, vol. 72, p. 015012, Jul. 2005.

[12] L. Younes, "Synchronous random fields and image restoration," IEEE Trans. Pattern Anal. Mach. Intell., vol. 20, no. 4, pp. 380-390, Apr. 1998.

[13] A. Graham, Nonnegative Matrices and Applicable Topics in Linear Algebra. John Wiley \& Sons, 1987.

[14] Wikipedia. Partition function (statistical mechanics). [Online]. Available: http://en.wikipedia.org/wiki/Partition_function_(statistical_mechanics)

[15] P. D. Lax, Linear Algebra and Its Applications, 2nd ed. John Wiley \& Sons, 2007. 